\title{
The Mental Health of People with Disabilities
}

\author{
Narsimulu $^{1 *}$
}

\section{ABSTRACT}

Disabled people are more likely to experience a lot, or a great deal, of worry than those who are not disabled. People with disabilities (e.g. physical impairments such as cerebral palsy, multiple sclerosis, spinal cord injury etc) are just as likely as the general population to experience mental health problems. They may be even more likely than the general population to need and use mental health services. Possible reasons for this may include - higher rates of poverty and unemployed amongst disabled people which are themselves associated with poor mental health; the greater risks of abuse experienced by disabled children and adults; and, some people with mental health support needs may be more likely to become physically disabled as a result of accidents or attempted suicide. There is also increasing acknowledgement that long-term mental health problems are correlated with conditions such as heart disease and diabetes.

People with disabilities appear to be at greater risk of mental health problems than the general population and therefore make a disproportionate contribution to mental health morbidity internationally. The personal and social costs of mental disorders are considerable throughout the world. The mental health of populations has been recognized as an international priority (World Health Organisation 2005). An important part of addressing this will be attending to the needs of people with disabilities who are a disproportionately disadvantaged group. In the following sections we briefly examine what is currently known about the association between disability and mental health. It is suggested that people with physical impairments and mental health support needs tend to be overlooked by policy-makers and commissioners of services. Many people with disabilities report having difficulty accessing mental health services because of their physical impairments. Many also have difficulty accessing physical disability services because of the inadequate recognition of mental health needs with disability related services.

\section{Keywords: Mental Health, Disabilities}

A Disability is a lack of ability relative to a personal or group standard or norm. In reality there is often simply a spectrum of ability. Disability may involve physical impairment, sensory

\footnotetext{
${ }^{1}$ Research Scholar, Dept. of Psychology, Osmania University, Hyderabad, Telangana

*Responding Author

(C) 2016 I Narsimulu; licensee IJIP. This is an Open Access Research distributed under the terms of the Creative Commons Attribution License (http://creativecommons.org/licenses/by/2.0), which permits unrestricted use, distribution, and reproduction in any Medium, provided the original work is properly cited.
} 


\section{The Mental Health of People with Disabilities}

impairment, cognitive or intellectual impairment, mental disorder (also known as psychiatric or psychosocial disability), or various types of chronic disease. A disability may occur during a person's lifetime or may be present from birth. Disability can be classified into several different types.

A model by which illness or disability is the result of a physical condition, is intrinsic to the individual (it is part of that individual's own body), may reduce the individual's quality of life, and causes clear disadvantages to the individual. As a result, curing or managing illness or disability revolves around identifying the illness or disability, understanding it and learning to control and alter its course.

\section{Some World Facts Regarding Disabilities:}

- According to UNICEF, 30 per cent of street youths are disabled.

- In most OECD countries, women report higher incidents of disability than men.

- Around 10 per cent of the world's population, or 650 million people, live with a disability. They are the world's largest minority.

- Eighty per cent of persons with disabilities live in developing countries, according to the UN Development Programme (UNDP).

- Comparative studies on disability legislation shows that only 45 countries have antidiscrimination and other disability-specific laws.

- Women with disabilities are recognized to be multiply disadvantaged, experiencing exclusion on account of their gender and their disability.

- This figure is increasing through population growth, medical advances and the ageing process, says the World Health Organization (WHO).

- In countries with life expectancies over 70 years, individuals spend on average about 8 years, or 11.5 per cent of their life span, living with disabilities.

- The World Bank estimates that 20 per cent of the world's poorest people are disabled, and tend to be regarded in their own communities as the most disadvantaged.

- In the United Kingdom, 75 per cent of the companies of the FTSE 100 Index on the London Stock Exchange do not meet basic levels of web accessibility, thus missing out on more than $\$ 147$ million in revenue.

- Mortality for children with disabilities may be as high as 80 per cent in countries where under-five mortality as a whole has decreased below 20 per cent, says the United Kingdom's Department for International Development, adding that in some cases it seems as if children are being "weeded out".

- Women and girls with disabilities are particularly vulnerable to abuse. A small 2004 survey in Orissa, India, found that virtually all of the women and girls with disabilities were beaten at home, 25 per cent of women with intellectual disabilities had been raped and 6 per cent of disabled women had been forcibly sterilized.

- Disability rates are significantly higher among groups with lower educational attainment in the countries of the Organisation for Economic Co-operation and Development 


\section{The Mental Health of People with Disabilities}

(OECD), says the OECD Secretariat. On average, 19 per cent of less educated people have disabilities, compared to 11 per cent among the better educated.

- For every child killed in warfare, three are injured and permanently disabled.

- In some countries, up to a quarter of disabilities result from injuries and violence, says WHO.

- Research indicates that violence against children with disabilities occurs at annual rates at least 1.7 times greater than for their non-disabled peers.

- Persons with disabilities are more likely to be victims of violence or rape, according to a 2004 British study, and less likely to obtain police intervention, legal protection or preventive care.

\section{Common Mental Health Issues Facing College Students}

Below is a list of serious mental health issues known to affect college students and young people: Depression: While it might be easy for a busy college student to write their depression off as school-induced stress, depressive tendencies can of course be symptoms of more serious mental health issues. In fact, a 2012 study reported that 44 percent of college students have one or more symptoms of depression. This startling statistic shows that hits depression faced by nearly half of all college students could lead to more complex mental health issues without the proper counseling to help them identify the source of their depression.

Anxiety: It goes without saying that most college students experience some degree of anxiety. As yo might expect, juggling assignments, exams, and part-time jobs can lead to serious levels of anxiety, which could then escalate into a major mental health issue or disorder. Students who feel like anxiety is getting the better of them should schedule some time to speak with a counsellor or mental health specialist in order to pinpoint the source of anxiety and figure out solutions to overcome it.

Suicide: The worst possible outcome of an untreated mental illness is suicide. Even for people without a serious mental disorder, the stress of an independent environment can lead to suicidal thoughts. Anyone who has seriously considered suicide should seek professional help immediately. Suicide hotlines staffed by specialists are usually the quickest and most discreet options for people to get the care and attention they need.

Bipolar Disorder: This is a major mental health disorder often characterized by extreme bouts of depression followed by periods of manic activity. With the stress and workload many college students face, it's easy to pass off symptoms of bipolar disorder as mood swings. According to WebMD, severe enough mood swings will interfere with a person's functioningcould be related to an underlying bi-polar disorder. Young people who find that their mood swings are causing difficulties in their personal or academic life should seek counseling from a mental health specialist immediately. 


\section{The Mental Health of People with Disabilities}

Eating Disorders: According to the National Eating Disorders Association, approximately 20 percent of women and 10 percent of men in college struggle with an eating disorder. For some, the pressure of losing weight and "looking good" might be enough to trigger the beginning of an eating disorder. For others, the stress of a busy social, academic and work schedule may make it difficult for them to find time to eat properly, which could also lead to a serious eating disorder down the line. While there are several different eating disorders, anorexia and bulimia are two of the most common. Eating disorders are serious and could lead to devastating consequences for a young person's health without immediate treatment from a mental health specialist.

Addiction: For individuals of any age, addiction can lead to significant and life threatening health issues without proper treatment. Addiction can be especially devastating for young people, who may turn to drugs, alcohol, or food to deal with general stress or an underlying mental health disorder. Binge drinking is an especially common form of addiction found on American campuses. According to the National Survey on Drug Use and Health, of the 61 percent of surveyed colleges students that drank, 40.5 percent binge drank and 16.3 percent were heavy drinkers. For many who struggle with addiction, often the hardest hurdle for them to overcome is admitting that they have a problem. If you or a young person you know is struggling with addiction, counselling from a mental health specialist or admission to a substance rehabilitation center are two viable treatment options.

Self-harm: Unlike other mental health issues, the underlying reason behind why young people choose to physically harm themselves still eludes researchers. Moreover, people who do harm themselves tend to do so in private and on areas of the body that may not be visible to others. Some estimate that up to 15 percent of college students have engaged in some form of selfharming behavior. Self-harm is a serious mental health issue that should be monitored by a trained mental health specialist.

Struggles with Identity: U.S. society has gradually come to accept the many disparate identities found within its borders. That said, in certain areas of the country, there is still a significant amount of intolerance directed towards people who identify themselves in a certain way. While a given identity will not necessarily indicate mental health struggles, the pressures of withstanding a hostile social environment could lead to severe stress and anxiety. Anyone struggling with extreme social pressures due to their lifestyle or identity should immediately seek help from a qualified specialist at their school or workplace.

\section{Improving the mental health of people with disabilities}

While there is clearly a need to address the physical health inequities experienced by people with mental disorders (Disability Rights Commission 2006; Rethink 2005), this chapter is concerned primarily with the mental health inequities faced by people with disabilities. These can be addressed through prevention initiatives and through the provision of mental health interventions that are appropriate for people who have other disabilities. 


\section{The Mental Health of People with Disabilities}

\section{Prevention}

There are two possible approaches to the promotion of mental health and the prevention of mental disorders in people with disabilities. The first is reducing the risk that people with disabilities will be exposed to conditions which are detrimental to mental health. The second is to improve the resilience of people with disabilities.

Evidence about the importance of socio-economic factors highlights a need for social and fiscal policies that reduce the chances of people with disabilities being exposed to the sorts of social conditions that negatively influence mental health (Emerson et al. 2009a). For example social policies to reduce income inequality could be expected to reduce the incidence and prevalence of both disability and mental disorders, and the association between the two. It is also important to ensure that policies facilitate employment for people with disabilities as unemployment contributes to financial hardship, social network contraction and psychological distress (Claussen 1999; Morrell et al. 1994). For those people with disabilities who are unable to work or unable to provide for themselves sufficiently through work, social protection should be provided at a level that is sufficient for people with disabilities and other vulnerable groups to live "flourishing" lives and participate in their communities (World Health Organisation 2008). Unfortunately even in higher income countries, income support payment rates for people with disabilities have failed to take account of the considerable extra costs associated with disability, resulting in people with disabilities facing high rates of poverty and hardship (Saunders 2006). Lastly, broad based policies that address such issues as public attitudes, disability discrimination, physical accessibility of community resources, and accessibility of information are needed to address disadvantages in social interaction and support and other elements of social exclusion for people with disabilities, such as exclusion from political engagement and civic participation (Burchardt et al. 2002). These types of prevention strategies address the need to understand mental health problems in vulnerable communities "less in terms of individual pathology and more as a response to relative deprivation and social injustice, which erode the emotional, spiritual and intellectual resources essential to psychological wellbeing" (Friedli 2009).

Prevention efforts aimed at improving individual resilience to environmental risk factors fall into three categories (Mrazek and Haggerty 1994). Universal interventions are directed at entire communities. Selective interventions target high-risk groups based on demographic characteristics, such as people with disabilities. Indicated interventions are aimed at individuals who have been identified as having sub-clinical symptoms or early signs but who do not yet fulfill the criteria for having a mental disorder.

We found no studies that specifically investigated the efficacy of programs for preventing mental health problems amongst people with disabilities. However there is some evidence for the effectiveness of prevention programs more generally (Vitaro and Tremblay 2008; WebsterStratton and Taylor 2001). For example, programs based on interpersonal psychotherapy and cognitive-behavioural therapy, particularly selective and indicated programs, have been found to 
be effective in preventing depression (Barrera et al. 2007; Cole 2008; Cuijpers et al. 2008; Gladstone and Beardslee 2009; Stice et al. 2009) and anxiety disorders (Bienvenu and Ginsburg 2007; Feldner et al. 2004). As an 'at risk' group, people with disabilities may benefit from such approaches. Further, evidence from studies with people with spinal cord injury and arthritis indicate that the coping strategies people with disabilities use can influence their mental health (Pollard and Kennedy 2007; Treharne et al. 2007), suggesting that coping skills training in particular may be helpful. As is discussed below, however, people with cognitive disabilities, such as those with intellectual disabilities or brain injury, may not respond to talking therapies without some modification of the methods used.

\section{Treating mental health problems}

For most people with disabilities there is no good reason to believe that generic evidence based treatments will be more or less efficacious than for people without disabilities. Perhaps for this reason, the treatment of mental health problems in people with disabilities has not been investigated. Investigations into mental health interventions for people with particular conditions are sparse and have not produced convincing evidence of the efficacy of particular treatments (e.g., Elliott and Kennedy 2004; Walker and Gonzalez 2007).

For people with intellectual disabilities, often considered among the most vulnerable and underserviced groups with regard to mental health (Yen et al. 2009), there is a lack of a strong evidence base to support interventions (Gustafsson et al. 2009; Hemmings 2008). For this group in particular, talking therapies, such as CBT and psychotherapy could not be expected to be used in the same way as for people without cognitive impairments. These approaches would be unsuitable for clients without enough verbal understanding and expressive abilities to engage in the necessary dialogue. For others, modifications may be required such as reducing the level of abstraction in conversations, shorter session times, and use of pictorial images such as time-lines. In psychoanalytic interventions, interpretive links should be made in smaller parts so that clients can retain what is being said, while for cognitive behavioural therapy aids to memory and concentration may be needed, such as flip charts, visual aids and role plays, with literacy based materials being adapted, such as by using tape recorders, dictaphones or simplified diaries with stickers (Beail and Jahoda, in press).

There is evidence internationally that high proportions of people who have mental disorders do not seek or receive mental health treatment (e.g., Australian Bureau of Statistics 2008; Costello et al. 2007; Freedenthal 2007). For people with disabilities, additional barriers such as physical access and a lack of understanding of mental health workers about disability issues may further reduce service usage. It is therefore important for mental health services to be "disability friendly". Further, disability workers and others coming into contact with people with disabilities should be proactive in liaising with and referring people with disabilities to mental health services rather than assuming that problems with adjustment to disability are inevitable or will improve with time. 


\section{Some Suggestions for Preventing the Stress and improve the mental health}

For college Disabled students and young people who do not struggle with significant mental health issues, there are still actionable steps to take in order to relieve stress and anxiety. Here are some suggestions to take into consideration:

Physical Activity: Physical activity releases "happy chemicals" in our brain known as endorphins that can have an almost immediate impact in balancing our mind and body's negative reaction toward stress and anxiety. Physical activity can also boost self-confidence and increase our ability to think clearly, focus, and inspire others to do the same.

Sleep and Diet Changes: Major changes in diet or sleep habits can also lead to elevated levels of stress or anxiety in a college student. Consistently staying up late to study for exams or finish assignments can mean some degree of sleep deprivation, which could lead to poor academic performance or more serious mental health issues. What's worse is that sleep deprivation and poor diet often go hand in hand. Dramatic shifts in diet will also impact a student's academic performance and mental health.

Psychiatric Care: If a student finds that the amount of stress they face is becoming too much to handle on their own, obtaining psychiatric care should be given serious consideration. Inpatient or outpatient care may be pursued depending on the severity of the mental health issues faced by the individual in need of care. Mental health specialists are there to help us overcome stress, anxiety, and many other issues impacting mental health. Remember that seeking psychiatric care should never be thought of as unreasonable.

Relaxation Exercises: No matter how stressed or anxious students become, there should always be something to count on as a source of positive relaxation. That said, many young people may not be able to find the opportunity to relax in the way that they prefer. Nonetheless, there are several quick and easy relaxation exercises to explore. Taking a few breaks each day to stretch, meditate, or even pick out a comfortable set of clothing can work to significantly reduce anxiety throughout the day.

Therapy and Counseling: Even if a young person feels that the stress in their life is not affecting their mental health, seeking therapy and counseling to understand how to better manage anxiety can still be helpful. Students may feel they have an exceptionally high tolerance for stress and anxiety, but that failing to learn new and better ways to manage their stress could rapidly lead to more serious mental health issues. Seeking therapy and counseling is often the safest and most effective way to get personalized advice before stress becomes a much more serious problem. 


\section{CONCLUSION}

People with disabilities are at greater risk of mental health problems than other members of the community. A multitude of factors appear to contribute to this association including the life consequences of disability, the poor health of people with mental disorders and the circular relationship that exists between disability, social exclusion and mental health problems. Mental health and disability awareness need to be integrated into social policy and health care delivery at all levels.

\section{REFERENCES}

American Psychiatric Association. 1994. Diagnostic and Statistical Manual of Mental Disorders. Washington (DC): APA.

Australian Bureau of Statistics. 2005. Australian Social Trends 2005. Canberra: ABS.

Australian Bureau of Statistics. 2008. Mental Health and Wellbeing Survey 2007. Canberra: ABS.

Australian Institute of Health and Welfare. 2008. Disability in Australia: Trends in prevalence, education, employment and community living. Canberra: AIHW.

Barrera AZ, Torres LD, Munoz RF. 2007. Prevention of depression: The state of the science at the beginning of the 21st century. International Review of Psychiatry 19(6):655-670.

Beail N, Jahoda A. in press. Working with people: Direct interventions. In: Emerson E, Hatton C, Bromley J, Caine A, Gone R, Dickson K, editors. Clinical Psychology and People with Intellectual Disabilities. 2nd ed. Chichester: Wiley.

Bienvenu OJ, Ginsburg GS. 2007. Prevention of anxiety disorders. International Review of Psychiatry 19(6):647-654.

Bruce ML, Hoff RA. 1994. Social and physical health risk factors for first-onset major depressive disorder in a community sample. Social Psychiatry and Psychiatric Epidemiology 29(4):165-171.

Burchardt T, Le Grand J, Piachaud D. 2002. Degrees of exclusion: Developing a dynamic, multidimensional measure. In: Hills J, Le Grand J, Piachaud D, editors. Understanding social exclusion. Oxford: Oxford University Press. p. 30-43.

Carroll LJ, Cassidy JD, Cote P. 2003. Factors associated with the onset of an episode of depressive symptoms in the general population. Journal of Clinical Epidemiology 56(7):651-658.

Cepeda ML, Allen FH, Cepeda NJ, Yang Y-M. 2000. Physical growth, sexual maturation, body image and sickle cell disease. Journal of the National Medical Association 92(1):10-14.

Chan J, Parmenter T, Stancliffe R. 2009. The impact of traumatic brain injury on the mental health outcomes of individuals and their family carers. AeJAMH (Australian e-Journal for the Advancement of Mental Health) 8(2):1-10.

Citrome, Yeomans J. 2005. Do guidelines for severe mental illness promote physical health and well-being? Psychopharmacol 19:102-109.

Claussen B. 1999. Alcohol disorders and re-employment in a 5-year follow-up of long-term unemployed. Addiction 94(1):133-138. 
Cole MG. 2008. Brief interventions to prevent depression in older subjects: A systematic review of feasibility and effectiveness. The American Journal of Geriatric Psychiatry 16(6):435443.

Cooper S-A, Smiley E, Morrison J, Williamson A, Allan L. 2007. Mental ill-health in adults with intellectual disabilities: prevalence and associated factors. British Journal of Psychiatry 190(1):27-35. 